\title{
Magneto-electric coupling in zigzag graphene nanoribbons
}

\author{
J. Jung 1, f $^{3}$ and A. H. MacDonald ${ }^{1}$ \\ ${ }^{1}$ Department of Physics, University of Texas at Austin, USA
}

\begin{abstract}
Zigzag graphene nanoribbons can have magnetic ground states with ferromagnetic, antiferromagnetic, or canted configurations, depending on carrier density. We show that an electric field directed across the ribbon alters the magnetic state, favoring antiferromagnetic configurations. This property can be used to prepare ribbons with a prescribed spin-orientation on a given edge.
\end{abstract}

Introduction - Expanding techniques that can achieve electrical control of spin is a key goal of both metal and semiconductor spintronics. [1, 2] In metal spintronics, for example, electrical spin-transfer torque[3] research seeks to amplify the potential[4] of technologies based on giant magnetoresistance [5] and tunnel magnetoresistance. [6] The aim of research on dilute magnetic semiconductors[7] is to create semiconductor materials in which magnetic properties are as sensitive to doping and external gate potentials as electrical properties. Recent interest in the spin Hall effect [8] and the topological magnetoelectric effect [9] is motivated by a search for effects which enable electrical control of spin in non-magnetic materials. In this context it is interesting to address the possibility of interesting magnetoelectric effects in graphitic material. The physics of zigzag graphene ribbons and edge terminations has received considerable attention recently $[10,11,12,13,14,15,16,17,18,19,20,21,22,23,24$, $25,26,27,28,29,30,31,32,33,34,35,36,37,38,39,40]$. Magnetism is expected in any graphitic material containing ribbon segments with zigzag edge $[10,11,12,13,14,15,16$, $17,18,19,20,21,22,23,24,25]$ terminations, for example highly defected bulk graphitic material. [41, 42, 43, 44] Thanks to progress in structural control of graphene flakes and related materials [45], prospects for mastering graphitic magnetism have improved. The perfect zigzag nano-ribbon studied in this Letter may be viewed as a model system in which graphitic magnetism is exhibited in its simplest and most essential form.

The magnetic ground state of a zigzag nanoribbon has collective moments localized near its edges. In the absence of a transverse electric field, a doped nanoribbon has either full or partial orientational alignment [46, 47] between moments on opposite edges, as illustrated schematically in Fig.[1]. We show that an external electric field applied across the ribbon can control the relative orientation angle $\theta$, and that this property can be used to prepare ribbons with a prescribed spinorientation on a given edge.

Hubbard Model Mean-Field Theory - Since ab initio densityfunctional and simpler model Hamiltonian approaches make essentially identical predictions [19, 23, 24, 43], we base our analysis of zigzag ribbon magnetism on a Hubbard model which allows the underlying physics to be identified more clearly. The success of the Hubbard model has been shown to be due to the essentially local character of edge magnetism in graphene ribbons [51]. The Hubbard model mean-field
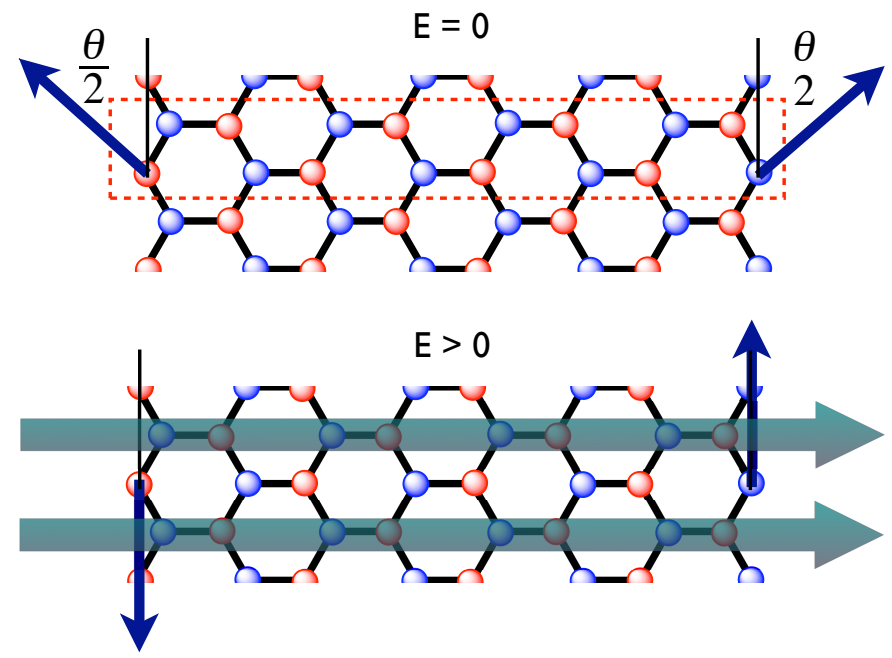

FIG. 1: Schematic representation of the influence of a lateral electric field on zigzag ribbon magnetism. Upper panel. Non-collinear ground state of a doped zigzag ribbon illustrating the angle $\theta$ between moment orientations on opposite edges. The red box indicates the unit cell of the ribbon. Lower panel. A lateral electric field drives the angle $\theta$ to $\pi$, an antiferromagnetic configuration similar to that of an undoped ribbon.

Hamiltonian,

$$
\begin{aligned}
H_{\sigma} & =-\gamma_{0} \sum_{\langle i, j\rangle} c_{i \sigma}^{\dagger} c_{j \sigma}+\sum_{i} c_{i \sigma}^{\dagger} c_{i \sigma}\left(v_{e x t}+e \mathscr{E} y_{i}\right) \\
& +\frac{U}{2} \sum_{i}\left[\left\langle c_{i \sigma}^{\dagger} c_{i \sigma}\right\rangle c_{i s}^{\dagger} c_{i s}-\left\langle c_{i \sigma^{\prime}}^{\dagger} \vec{\tau}_{\sigma^{\prime}, \sigma} c_{i \sigma}\right\rangle \cdot c_{i s^{\prime}}^{\dagger} \vec{\tau}_{s^{\prime}, s} c_{i s}\right](1)
\end{aligned}
$$

has a term which represents hopping between nearest neighbor $\pi$-orbitals with amplitude $\gamma_{0}=2.6 \mathrm{eV}$, an external potential term which accounts for the transverse electric field, and a mean-field interaction term. The operator $c_{i \sigma}^{\dagger}$ creates a $\pi$ orbital electron at site $i$ with spin $\sigma, \mathscr{E}$ is the transversal electric field, $y_{i}$ is the position of lattice site $i$ along the ribbon width and $\vec{\tau}_{\sigma^{\prime}, \sigma}$ represents the elements of the three Pauli matrixes. As a convenience we include a constant term $v_{e x t}=-U$ in the external potential which removes the interaction with a unit charge on each site from the mean-field quasiparticle energy. (All spin indices in Eq. [1] are summed over.) Note that the mean-field interaction energy of an electron on site $i$ is spin-dependent and proportional to the density of opposite spin electrons. Following Yazyev et al.,[43] we choose $U=3 e V$ a value slightly larger than estimates based on the 
local density approximation used in some previous Hubbard model analyses [19, 21, 47]. We have used $1200 k$-points in the Brillouin zone for the self-consistent calculations. Zig-zag edge magnetism is very sensitive[47] to the net charge density of the ribbon $\delta n$, which we refer to as doping whether due to chemical dopants or gate voltages and measure per repeat distance $a=2.46 \AA$ along the edge. The corresponding areal density is $\delta n_{2 D}=\delta n / W$ where the ribbon width $W=\sqrt{3} N a / 2$ and $N$ is the number of atom pairs per ribbon unit cell.
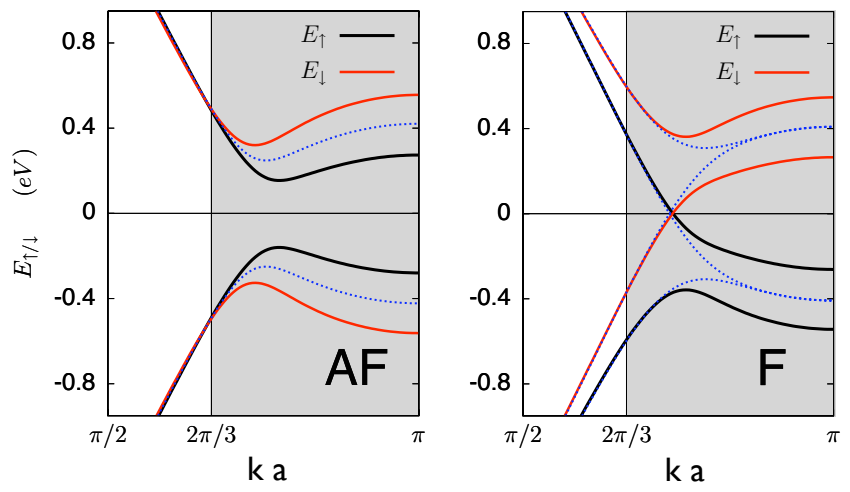

FIG. 2: Edge state bands of a zigzag ribbon with $N=20$, nearestneighbor hopping $\gamma_{0}=2.6 \mathrm{eV}$ and on site repulsion $U=3 \mathrm{eV}$ under a transverse electric field of $\mathscr{E}=0.2 \mathrm{eV} / \mathrm{nm}$. Left panel: In the AF case high-spin (see text) bands shift toward the Fermi energy and the low-spin bands move away. The wavefunctions of the occupied bands shift density to the low-energy edge. Right panel: In the F case $\Delta_{\mathscr{E}}$ shifts the wavevector at which majority and minority spin bands cross to slightly larger values of $|k|$. The dotted lines show the bands at $\Delta_{\mathscr{E}}=0$.

Edge-Only Model-The influence of a transverse electric field on electronic structure in neutral ribbons has been studied previously for both armchair [48] and zigzag cases [16, 49, 50] using density functional theory. The essentials of zigzagribbon magnetism and of the transverse-field magnetoelectric effect are captured by an edge-state-only model[19, 21]; the qualitative discussion below refers mainly to this model and to the special case of collinear magnetic states $(\theta=0$ or $\theta=\pi)$, but the numerical calculations and the phase-diagram results are based on solutions of the the full non-collinear $\pi$ band Hubbard model self-consistent field equations. In the edge-only model the spin-dependent mean-field Hamiltonian for collinear states takes the form [21, 51]

$$
H_{\sigma}(k)=\left(\sigma \Delta_{z}(k)+\Delta_{\mathscr{C}}(k)\right) \tau_{z}+\left(\Delta_{0}(k)+h_{z}\right) \sigma I+t(k) \tau_{x} .
$$

Here the $\tau_{\alpha}$ are Pauli matrices which act on the which edge degree of freedom. The terms proportional to $\tau_{z}$ in Eq. [2] therefore represent the difference in energy between left $\left(\tau_{z} \rightarrow 1\right)$ and right $\left(\tau_{z} \rightarrow-1\right)$ edges for electrons of spin $\sigma$, whereas the

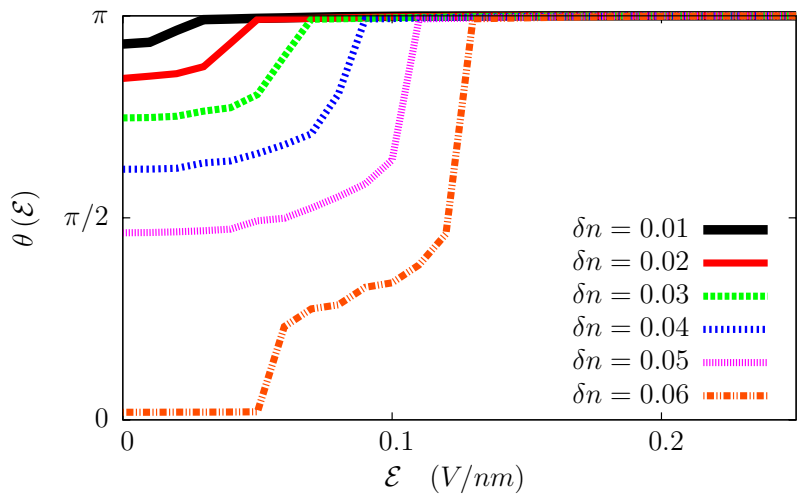

FIG. 3: Inter-edge relative spin orientation angle $\theta$ evaluated on the outermost edge atoms as a function of transverse electric field $\Delta_{\mathscr{E}}$ for a series of doping $\delta n$ values. For $\Delta_{\mathscr{E}}=0$ doping leads immediately to a canting angle $\theta<\pi$ and eventually for $\delta_{n}$ larger than $\sim 0.05$ to a ferromagnetic state with $\theta=0$. A finite $\Delta_{\mathscr{E}}$ favors the AF state as explained in the text and drives $\theta$ toward its undoped value.

term proportional to $\tau_{x}$ represents the momentum-dependent inter-edge hopping amplitude. Zig-zag edge magnetism follows from the property [21] that $t(k)$ vanishes rapidly with ribbon width in the part of the Brillouin-zone $(2 \pi / 3 a<|k|<$ $\pi / a)$ in which edge states reside. In Eq. (2), $\Delta_{z}(k)$ captures the difference between exchange energies on opposite edges, which vanishes in the $\theta=0(\mathrm{~F})$ state in the absence of a transverse field, whereas $\Delta_{0}(k)$ captures the spin-dependence of the edge average, which vanishes in the $\theta=\pi$ (AF) state. (An irrelevant spin and edge independent exchange energy has been dropped from $H_{\sigma}$.) Both exchange energies are large only for the edge-states $(|k|>2 \pi / 3 a)$. $h_{z}$ accounts for Zeeman coupling to the ribbon spins by an external magnetic field when present.

Magnetoelectric Coupling in Undoped Ribbons- The eigenenergies of this Hamiltonian are

$$
E_{\sigma}^{ \pm}(k)=\sigma\left(\Delta_{0}(k)+h_{z}\right) \pm \sqrt{\left(\sigma \Delta_{z}(k)+\Delta_{\mathscr{E}}(k)\right)^{2}+t^{2}(k)} .
$$

Note that there are always four distinct eigenvalues in the ferromagnetic case, whereas the antiferromagnetic state bands occur in doubly-degenerate pairs when $\Delta_{\mathscr{E}} \rightarrow 0$. For undoped ribbons the lowest two edge states bands are normally fully occupied. In Fig.2 we plot ribbon band structures for both $\mathrm{AF}$ and $\mathrm{F}$ states of a neutral ribbon calculated using a constant transverse electric field of $\mathscr{E}=0.2 \mathrm{~V} / \mathrm{nm}$. States that are shifted down (up) in energy relative to the $\Delta_{\mathscr{E}}=0$ case are localized on the low (high) potential side of the ribbon. (Note that a constant field generates a $k$-dependent $\Delta_{\mathscr{E}}$ because of the $k$-dependence of the degree of edge state localization.) In the F-state the Fermi energy is pinned to a band-crossing near $|k|=2 \pi / 3 a$ between the higher energy majority spin band and the lower energy minority spin band. In the AF case we refer to the spin-orientation which dominates occupied states on the low (high) potential side of the ribbon as the low-spin (highspin). (In Fig.2, $\downarrow$ is the low-spin.) A transverse spin shifts 


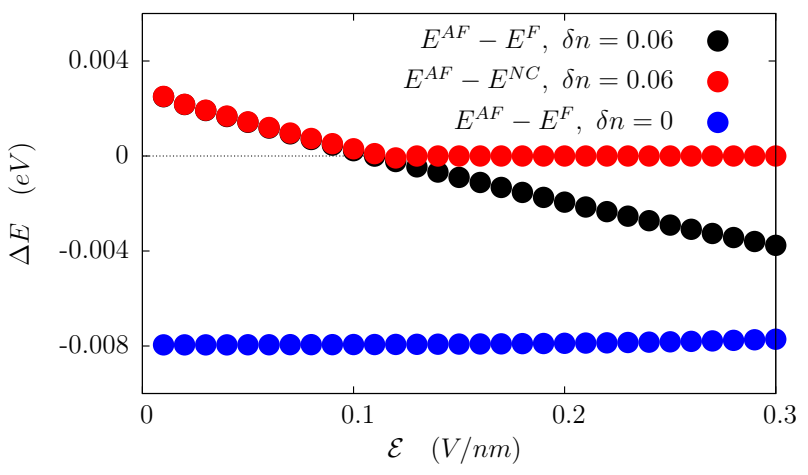

FIG. 4: Energy differences (per ribbon unit cell) between the spin collinear AF, F solutions and, for finite-doping, the minimum energy non-collinear (NC) solution. The energy difference between $\mathrm{AF}$ and $\mathrm{F}$ solutions for $\delta n=0$ has a weak transverse electric field dependence.

the energies of both occupied and unoccupied high-spins toward the Fermi level, lowering the gap. A sufficiently large transverse field will close the indirect gap, creating a halfmetallic band structure with only high-spin bands crossing the Fermi level. This is the magnetoelectric effect discussed in earlier [16, 22, 50] work. The energy difference between $\mathrm{F}$ and AF states [21] is relatively unchanged by a transverse field. Below we show that in doped ribbons a transverse field tilts the competition between $\mathrm{F}$ and AF states in favor of the latter, yielding a distinct and stronger magnetoelectric effect. Magneto-electric coupling at finite doping - We consider for definiteness the case of n-type ribbons in which carriers are added by gate doping. In the absence of a transverse electric field, doping favors [46, 47] the gapless F state over the gapful $\mathrm{AF}$ state. The transition between undoped $\mathrm{AF}$ and doped $\mathrm{F}$ states occurs continuously by varying the relative orientation angle $\theta$ between its $\operatorname{AF}(\theta=\pi)$ and $\mathrm{F}(\theta=0)$ end points. The effect we discuss in this paper is based on the following simple observation concerning the edge-state bands plotted in Fig. 2. In the AF case the conduction band states which are occupied upon doping are high-spin antibonding states, which are localized on the low-energy side of the ribbon. For the F state, on the other hand, there are occupied states in two bands, one localized on the high-energy side and one localized on the lowenergy side. The net effect is that a transverse field favors the AF state in doped zigzag ribbons. In Fig. 3 we plot the relative angle between spin polarizations on opposite edges $v s$. lateral electric field for a series of different doping values. These results were obtained by non-collinear spin self-consistent field calculations and confirm the expected magnetoelectric effect. In Fig. 4 we compare the transverse field dependence of the energy difference between $\mathrm{F}$ and $\mathrm{AF}$ states for doped and undoped systems. The electric field strength required to convert $\mathrm{F}$ states into AF states in doped ribbons is much smaller than the field required to close the AF-state gap in the undoped case.

Discussion - Typical results for the edge-state bands of both
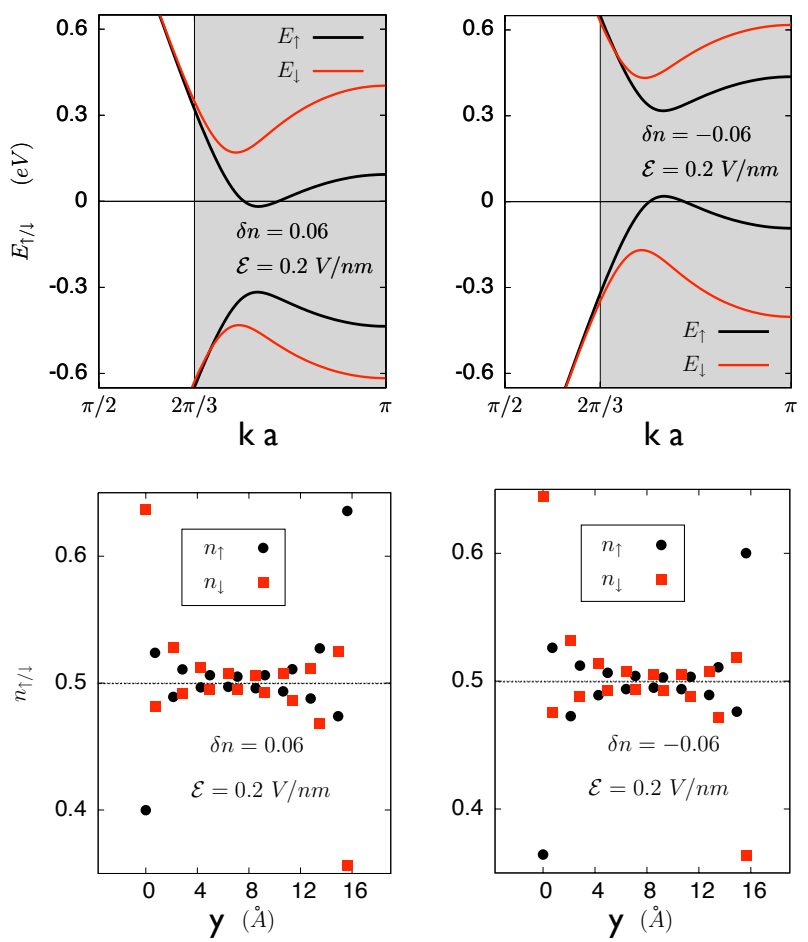

FIG. 5: Top panel: Band structure of $n$ and $p$ gate-doped $N=8$ zigzag graphene nanoribbons in the presence of a transverse electric field, $\mathscr{E}=0.2 \mathrm{~V} / \mathrm{nm}$, strong enough to produce a collinear $\theta=\pi$ ground state. Note that the edge-state bands are half-metallic in both cases. Bottom panel: Spin resolved occupation $n_{\uparrow / \downarrow}(y)$ across the robbon: majority spin electrons ( $\sigma=\uparrow$ for $n$-doped and $\sigma=\downarrow$ for $p$ doped) in this figure accumulate on the high potential edge of the ribbon for $\delta n=0.06$ and on the low-potential side for $\delta n=-0.06$.

electron and hole doped zigzag ribbons with a transverse field strong enough to induce the $\theta=\pi$ state are illustrated in Fig.5. Because of the partial occupation of the highest unoccupied band of the $n$-doped case and the lowest unoccupied band in the $p$-doped case, the $\theta=\pi$ state has an overall spinpolarization proportional in magnitude to the doping. Note that these $\theta=\pi$ states are always half-metallic. This ferromagnetic component of the order allows Zeeman coupling from an external magnetic field to fix the spin-orientation on each edge. It follows from Fig.5 that the majority spins are high-energy spins in the $n$-doped case and low-energy spins in the $p$-doped case. For a fixed magnetic field direction, the spin-orientations on both edges can therefore be switched with a gate voltage, which changes the sign of the carrier density. This remarkable property of zigzag edge magnetism has no parallel of which we are aware in any other magnetic system.

In closing we remark that the one-dimensional character of zigzag edge magnetism works against robust collective spin properties. As analyzed in more detail elsewhere 18 , 22], the consequences of reduced dimensionality are somewhat mitigated by the the substantial stiffness [18, 22] of zigzag edge moments. Nevertheless, robust magnetism in graphitic nanostructures will likely require exchange-coupled 
two-dimensional ribbon networks. The rather unique properties of graphitic magnetism discussed in this Letter motivate an effort to realize structures of this type.

Acknowledgement. We acknowledge financial support from the Welch Foundation, NRI-SWAN, the DOE, the Spanish Ministry of Education through the MEC-Fulbright program and Fan Zhang for his help with figures.

* Electronic address: jeil@ physics.utexas.edu

[1] S. A. Wolf et al. Science 294, 1488 (2001). I. Zutic et al. Rev. Mod. Phys. 76, 323 (2004). T. Dietl et al. "Spintronics", Elsevier (2008).

[2] D. Awschalom and N. Samarth, Physics 2, 50 (2009).

[3] D. C. Ralph, M. D. Stiles J. Magnetism and Magnetic Materials 320, 1190 (2008).

[4] J. Akerman, Science 308, 508 (2005).

[5] P. Grünberg, Rev. Mod. Phys. 80, 1531 (2008).

[6] M. Julliere, Phys. Lett. 54A, 225 (1975). J. Mathon and A. Umerski, Phys. Rev. B 63, 220403 (2001).

[7] H. Munekata, et al. Phys. Rev. Lett. 63, 1849 (1989). H. Ohno et al. Nature 408, 944946 (2000). A. H. MacDonald et al. Nature Materials 4, 195-202 (2005). T. Jungwirth et al, Rev. Mod. Phys. 78, 809 (2006).

[8] J.E. Hirsch, Phys. Rev. Lett. 83: 1834 (1999). Sinova et al. Phys. Rev. Lett. 92, 126603 (2004). S. Murakami et al., Science 301, 1348 (2003).

[9] X.-L. Qi et al. Phys. Rev B. 78, 195424 (2008).

[10] M. Fujita, K. Wakabayashi, K. Nakada, K. Kusakabe, J. Phys. Soc. Jpn. 65, 1920 (1996).

[11] T. Hikihara, X. Hu, H.-H. Lin, and C.-Y. Mou Phys. Rev. B 68, 035432 (2003).

[12] S. Dutta, S. Lakshmi, and S. K. Pati, Phys. Rev. B 77, 073412 (2008).

[13] H. Lee, Y.-W. Son, N. Park, S. Han, and J. Yu, Phys. Rev. B 72, 174431 (2005).

[14] K.-I. Sasaki, S. Murakami, R. Saito, J. Phys. Soc. Jpn. 75, 074713 (2006).

[15] Y.-W. Son, Marvin L. Cohen, and Steven G. Louie, Phys. Rev. Lett. 97, 216803 (2006).

[16] Y.-W. Son, Marvin L. Cohen, and Steven G. Louie, Nature 444, 347 (2006).

[17] L. Pisani, J. A. Chan, B. Montanari, and N. M. Harrison Phys. Rev. B 75, 064418 (2007).

[18] O. V. Yazyev, M. I. Katsnelson Phys. Rev. Lett. 100, 047209 (2008).

[19] J. Fernández-Rossier, Phys. Rev. B 77, 075430 (2008).

[20] W. Y. Kim and K. S. Kim, Nature Nanotechnology 3, 408 (2008).

[21] J. Jung. T. Pereg-Barnea, A. H. MacDonald, Phys. Rev. Lett.
102,227205 (2009).

[22] J.-W. Rhim and K. Moon, Phys. Rev. B 80, 155441 (2009).

[23] S. Bhowmick and V. B. Shenoy, J. Chem. Phys. 128, 244717 (2008).

[24] J. Fernández-Rossier, J. J. Palacios Phys. Rev. Lett. 99, 177204 (2007).

[25] M. Topsakal, H. Sevincli, S. Ciraci, App. Phys. Lett. 92, 173118 (2008)

[26] W. Yao, S. Yang, Q. Niu, Phys. Rev. Lett. 102, 1 (2009).

[27] K. Nakada, M. Fujita, G. Dresselhaus, M. S. Dresselhaus, Phys. Rev. B 54, 17954 (1996).

[28] K. Wakabayashi, M. Fujita, H. Ajiki, M. Sigrist, Phys. Rev. B 59, 8271 (1999).

[29] M. Ezawa, Phys. Rev. B 73, 045432 (2006).

[30] L. Brey and H. A. Fertig, Phys. Rev. B 73, 235411 (2006).

[31] D. Gunlycke, D. A. Areshkin, L. Junwen, J. W. Mintmire, C. T. White, Nano letters. 7, 3608 (2007); D. Gunlycke, H. M. Lawler, C. T. White, Phys. Rev. B. 75, 29-33 (2007).

[32] M. Zarea, C. Busser and N. Sandler, Phys. Rev. Lett. 101, 196804 (2008).

[33] M. Y. Han, Barbaros Özyilmaz, Y. Zhang, and P. Kim, Phys. Rev. Lett. 98, 206805 (2007).

[34] X. Li, X. Wang, L. Zhang, S. Lee, H. Dai, Science 319, 1229 (2008).

[35] S. S. Datta, D. R. Strachan, S. M. Khamis and A. T. C. Johnson, Nano Lett. 81912 (2008).

[36] L. C. Campos, V. R. Manfrinato, J. D. Sanchez-Yamagishi, J. Kong, P. Jarillo-Herrero, Nano letters. 9, 2600 (2009)

[37] L. Ci, L. Song, D. Jariwala, et al. Adv. Mat. 21, 4487 (2009)

[38] X. Jia, M. Hofmann, V. Meunier, et al. Science 323, 1701 (2009)

[39] C.O. Girit, J. C. Meyer, R. Erni, et al. Science 323, 1705 (2009)

[40] A. Chuvilin, J. C. Meyer, G. Algara-Siller, U. Kaiser, New Journal of Physics 11, 083019 (2009)

[41] J. J. Palacios, J. Fernández-Rossier, and L. Brey Phys. Rev. B 77, 195428 (2008).

[42] H. Ohldag et al., Phys. Rev. Lett. 98, 187204 (2007).

[43] O. V. Yazyev, Phys. Rev. Lett. 101, 037203 (2008).

[44] J. S Cervenka, M. I. Katsnelson and C. F. J. Flipse, Nature Physics 5, 840 (2009)

[45] A. H. Castro Neto, F. Guinea, N. M. R. Peres, K. S. Novoselov and A. K. Geim, Rev. Mod. Phys. 81, 109 (2009); A. K. Geim and K. S. Novoselov et al., Nature Materials 6, 183 (2007); A. K. Geim and A. H. MacDonald, Physics Today 60, 35 (2007).

[46] K. Sawada, F. Ishii, M. Saito, S. Okada, and T. Kawai, Nano Lett. 9, 269 (2009).

[47] J. Jung and A. H. MacDonald, Phys. Rev. B 79, 235433 (2009).

[48] D. S. Novikov, Phys. Rev. Lett. 99, 056802 (2007).

[49] E. Rudberg, P. Salek, and Y. Luo, Nano Lett., 7, 2211 (2007).

[50] Er-Jun Kan et al. Appl. Phys. Lett. 91, 243116 (2007).

[51] J. Jung, T. Pereg-Barnea and A. H. MacDonald, to be submitted. 